\title{
Medical trainees in research-focused programs at higher risk for depression
}

\author{
— Cite as: CMAJ 2019 February 25;191:E240. doi: 10.1503/cmaj.109-5716
}

Posted on cmajnews.com on Feb. 5, 2019.

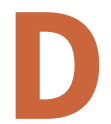

octors face alarming risks of burnout, depression and suicide, and tend to be most vulnerable during residency. A recent study suggests some residency programs put trainees at greater risk of depression than others.

Published in Academic Medicine, the study found that trainees reported increased symptoms of depression in programs with long working hours, poor faculty feedback and inpatient learning experiences, and higher research rankings. According to the authors, "these factors are potentially valuable targets for intervention to improve the wellness and mental health of residents."

The researchers surveyed more than 1200 internal medicine interns from 54 programs across the United States at regular intervals before and during residency. Trainees reported increasing symptoms of depression as training progressed. On average, one in three met the criteria for major depression. However, some programs had high rates of depression while others had low rates.

Quality of faculty feedback was the strongest predictor of these differences, even after accounting for individual factors that could make residents prone to depression. Trainees in programs with higher research rankings, as measured by the number of papers published and grants won by alumni, also reported increased symptoms of depression. Notably, there was no link between trainees' baseline risk for depression and a program's research ranking, ruling out the possibility that higher-ranking programs selected trainees who were predisposed to depression. And despite wide variation among programs, rates of depression within a given program were relatively consistent.

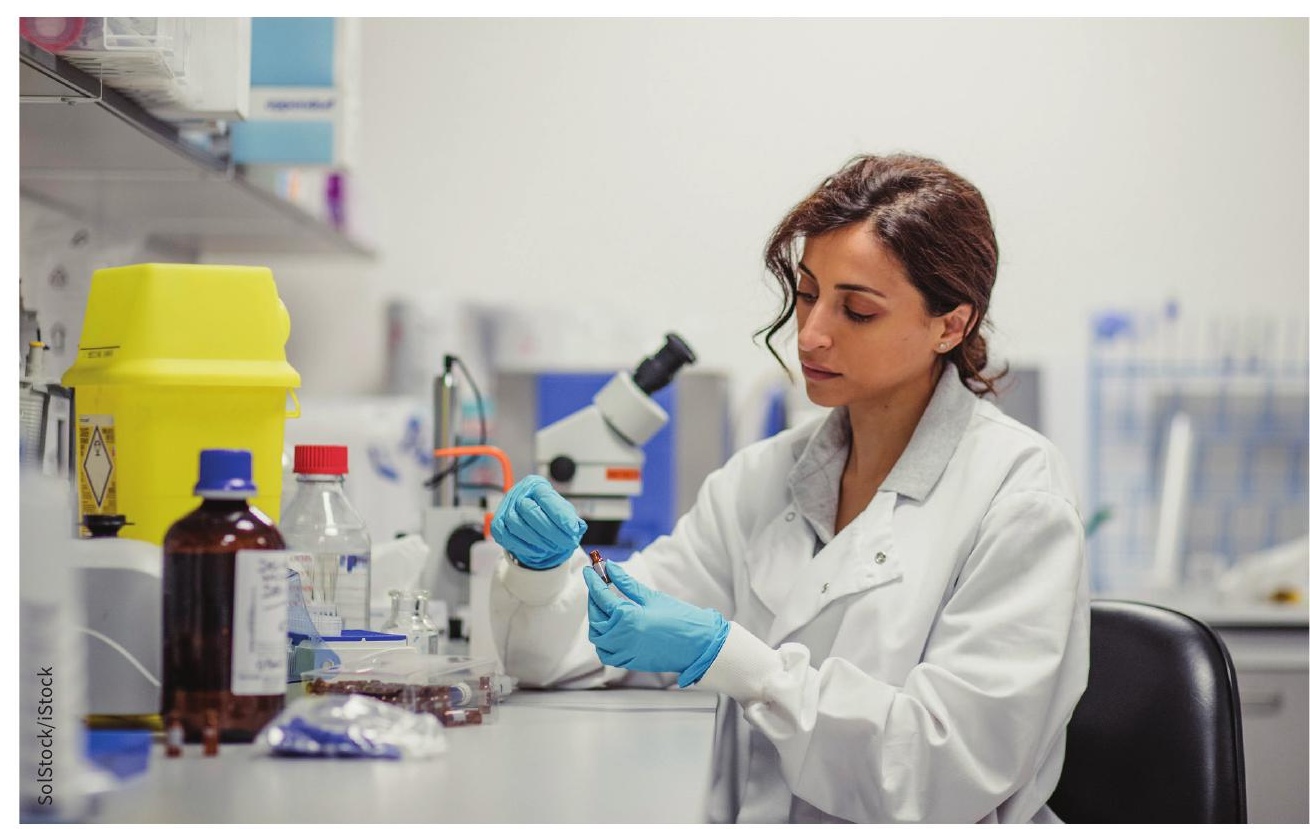

Medical trainees in research-focused programs may be under pressure to achieve both clinically and in scholarly pursuits.

The authors suggested that high pressure to achieve both clinical and scholarly excellence may contribute to trainee depression. Research ranking may also be a proxy for other stressors, such as the competitive culture of a program or the complexity of patients seen by residents. More studies are needed to understand these relationships, the authors wrote.

The study has several important limitations, including its focus on internal medicine residents and reliance on trainees' own assessments of their symptoms and working environments. Even so, the authors concluded, "these findings suggest that the residency program environment plays a central role in the mental health of medical interns."

It's a view that's slowly gaining traction in Canada. At an international phys- ician health conference hosted in Toronto last year, medical leaders called on universities to make wellness a core competency of medicine to kickstart faculty development and training reforms.

Compared to other physicians in Canada, residents face a $48 \%$ higher risk of burnout, 95\% higher risk of depression and $72 \%$ higher risk of suicidal thoughts. More than three-quarters of those surveyed by Resident Doctors of Canada reported experiencing harassment and intimidation on the job. Almost all were on the receiving end of inappropriate comments. Although patients were the most common source of abuse, more than half reported harassment and intimidation coming from staff physicians and other health professionals.

\section{Lauren Vogel, CMAJ}

